\title{
EFLDO sensitizes liver cancer cells to TNFSF10-induced apoptosis in a p53-dependent manner
}

\author{
YANBO QU ${ }^{1}$, ZHIXIN LIAO ${ }^{1,2}$, XINZHU WANG ${ }^{1}$, JING ZHANG $^{1}$ and CHAO LIU ${ }^{3}$ \\ ${ }^{1}$ Department of Pharmaceutical Engineering, School of Chemistry and Chemical Engineering, Southeast University, Nanjing, \\ Jiangsu 211189; ${ }^{2}$ Jiangsu Province Hi-Tech Key Laboratory for Biomedical Research, Southeast University, Nanjing, \\ Jiangsu 211189; ${ }^{3}$ Key Laboratory of Agro-Products Processing Technology of Shandong Province, Institute of Agro-Food \\ Science and Technology, Shandong Academy of Agricultural Sciences, Jinan, Shandong 250100, P.R. China
}

Received June 18, 2018; Accepted March 6, 2019

DOI: $10.3892 / \mathrm{mmr} .2019 .10046$

\begin{abstract}
Ent-3 $\alpha$-formylabieta-8(14),13(15)-dien-16,12 $\beta$-olide (EFLDO) is a compound extracted from Euphorbia lunulata Bge exhibiting anti-proliferative activity in vitro. In the present study, EFLDO was identified to sensitize HepG2 cells to tumor necrosis factor (TNF) superfamily member 10 (TNFSF10)-induced apoptosis. Liver cancer cells were resistant to TNFSF10; however, EFLDO increased TNFSF10-induced cancer cell viability inhibition and cell apoptosis induction as assessed by MTT assay and Annexin V-fluorescein isothiocyanate (FITC)/propidium iodide assay, respectively. The western blotting results suggested that treatment with EFLDO increased TNFSF10-induced upregulation of the protein expression levels of pro-apoptotic proteins, including BCL2 associated agonist of cell death, BCL2 associated X, apoptosis regulator, caspase-3 (CASP3) and CASP8. Furthermore, treatment with EFLDO increased TNFSF10-mediated downregulation of the protein expression level of the anti-apoptotic protein BCL2 apoptosis regulator. Notably, the increase in the activity of CASP3 was consistent with the western blotting results. Treatment with EFLDO sensitized liver cancer cells to TNFSF10, and apoptosis was induced via the upregulation of TNF receptor superfamily member 10a (TNFRSF10A) and TNFRSF10B in a tumor protein p53 (p53)-dependent manner, as detected by
\end{abstract}

Correspondence to: Professor Zhixin Liao, Department of Pharmaceutical Engineering, School of Chemistry and Chemical Engineering, Southeast University, 2 Southeast University Road, Nanjing, Jiangsu 211189, P.R. China

E-mail: zxliao@seu.edu.cn

Professor Chao Liu, Key Laboratory of Agro-Products Processing Technology of Shandong Province, Institute of Agro-Food Science and Technology, Shandong Academy of Agricultural Sciences, 202 Gongyebei Road, Jinan, Shandong 250100, P.R. China E-mail: liuchao555@126.com

Key words: liver cancer, ent-3 $\alpha$-formylabieta-8 (14),13 (15)-dien$16,12 \beta$-olide, tumor necrosis factor superfamily member 10 , p53-dependent way reverse transcription-quantitative polymerase chain reaction and western blot analyses. In addition, p53 was identified to be necessary for EFLDO-induced sensitivity to TNFSF10, as assessed by western blotting and Annexin V-FITC assay. Collectively, the present results suggested a novel mechanism underlying EFLDO function in liver cancer. Treatment with EFLDO was able to increase the antitumor effect of TNFSF10 in liver cancer cells in a p53-dependent manner.

\section{Introduction}

Liver cancer is the fifth most common malignant tumor, and exhibits the second highest mortality rate among malignant tumors (1-3). Liver cancer has a high incidence rate, and accounts for $\sim 55 \%$ of the cancer incidence worldwide (4). Additionally, the majority of patients with liver cancer at an advanced stage have a poor prognosis (5). Therefore, it is necessary to identify novel drugs to overcome the chemotherapeutic resistance of liver cancer cells and to develop novel treatments for patients with liver cancer.

Tumor necrosis factor (TNF) superfamily member 10 (TNFSF10) belongs to the TNF family, and is able to induce cell apoptosis in various types of tumor cells; however, its pro-apoptotic activity has not been detected in normal healthy cells (6-9). Cell apoptosis may be initiated by the interaction between TNFSF10 and TNF receptor superfamily member 10a (TNFRSF10A)/TNFRSF10B (10,11). Subsequently, caspase-8 (CASP8) is recruited to the death-inducing signaling complex and may cause the activation of CASP3, inducing cell apoptosis (12-14). TNFSF10 resistance may be acquired via multiple molecular mechanisms, including the differential expression of death receptors and the increased expression of anti-apoptotic factors (15). Notably, treatments combining TNFSF10 with various anticancer drugs have been demonstrated to improve the anticancerogenic effects of a number of compounds (16).

Due to the antiproliferative activity exhibited by ent- $3 \alpha$-form ylabieta-8(14),13(15)-dien-16,12 $\beta$-olide (EFLDO) in MCF-7 cells and NCI-H460 cells (17), EFLDO has attracted the interest of various research studies (18). The aim of the present study was to investigate the antitumor activity of EFLDO and the potential molecular mechanisms underlying EFLDO function in promoting the inhibitory effects of TNFSF10 in liver cancer cells. 


\section{Materials and methods}

Cell culture. HepG2 cells (19) and non-cancerous normal liver LO2 cells were purchased from The American Type Culture Collection (Manassas, VA, USA). The cells were cultured in Dulbecco's modified Eagle's medium (DMEM; HyClone; GE Healthcare Life Sciences, Logan, UT, USA), supplemented with $10 \%(\mathrm{v} / \mathrm{v})$ fetal bovine serum (HyClone; GE Healthcare Life Sciences) and $1 \%$ penicillin-streptomycin (HyClone; GE Healthcare Life Sciences) in an incubator with $5 \% \mathrm{CO}_{2}$ at $37^{\circ} \mathrm{C}$.

Drug treatments. Non-cancerous normal liver LO2 cells were treated with EFLDO $(3 \mu \mathrm{M})$ and/or TNFSF10 $(25 \mathrm{ng} / \mathrm{ml}$, Thermo Fisher Scientific, Inc., Waltham, MA, USA), and cultured in an incubator with $5 \% \mathrm{CO}_{2}$ at $37^{\circ} \mathrm{C}$ for $24 \mathrm{~h}$. EFLDO was extracted from Euphorbia lunulata Bge as previously described (17). Hepatoblastoma cells were treated with EFLDO $(3 \mu \mathrm{M})$, TNFSF10 $(25 \mathrm{ng} / \mathrm{ml})$, caspase inhibitor z-DEVD-FMK (working concentration, $40 \mu \mathrm{M}$; R\&D Systems, Inc., Minneapolis, MN, USA; cat. no. FMK004) and/or the p53 inhibitor pifithrin- $\alpha$ (PFT- $\alpha$; working concentrations, 20 and $40 \mu \mathrm{M}$; Sigma-Aldrich; Merck KGaA, Darmstadt, Germany) and cultured in an incubator with $5 \% \mathrm{CO}_{2}$ at $37^{\circ} \mathrm{C}$ for $24 \mathrm{~h}$ prior to further experimentation.

MTT assay. Hepatoblastoma HepG2 cells and non-cancerous normal liver LO2 cells were seeded into a 96-well plate at a density of $3 \times 10^{3}$ cells/well. Following addition of the indicated drug into the culture medium, the cells were incubated in an incubator with $5 \% \mathrm{CO}_{2}$ at $37^{\circ} \mathrm{C}$ for $24 \mathrm{~h}$. Subsequently, the cell viability was measured using the MTT assay at $570 \mathrm{~nm}$. The MTT assay was performed using DMSO (Sigma-Aldrich; Merck $\mathrm{KGaA}$ ) as the solvent to dissolve the formazan as previously described (20).

Annexin V-fluorescein isothiocyanate (FITC) assay. The Annexin V-FITC assay (Becton, Dickinson and Company, Franklin Lakes, NJ, USA) was performed to quantify the percentage of apoptotic cells, according to the manufacturer's protocol. HepG2 cells were incubated for $24 \mathrm{~h}$, collected, washed twice with PBS and incubated in $1 \mathrm{X}$ binding buffer (provided in the Annexin V-FITC kit). Subsequently, Annexin V-FITC and propidium iodide (PI) were added to the cells and cells were incubated for $20 \mathrm{~min}$ at room temperature in the dark. Following incubation with Annexin V-FITC and PI, the cells were analyzed using a flow cytometer (Becton, Dickinson and Company) and the results were analyzed using the CellQuest Pro software (version 5.1; Becton, Dickinson and Company).

CASP3 Activity assay. CASP3 activity was measured using the CASP3 activity assay kit (Chemicon International; Thermo Fisher Scientific, Inc.) according to the manufacturer's protocol. HepG2 cells were lysed using lysis buffer provided in the kit on ice for $10 \mathrm{~min}$ and centrifuged for $5 \mathrm{~min}$ at $12,000 \mathrm{x} \mathrm{g}$ at $4^{\circ} \mathrm{C}$. Caspase substrate solution was added to the supernatant and incubated for $2 \mathrm{~h}$ at $37^{\circ} \mathrm{C}$, subsequently, the activity was measured using an ELISA plate reader at $405 \mathrm{~nm}$.

Western blot. HepG2 cells were incubated with DMEM for $48 \mathrm{~h}$. Cells at a confluency of $70 \%$ were lysed using radioimmunoprecipitation assay buffer (Beyotime Institute of Biotechnology, Haimen, China) containing a protease inhibitor cocktail (Roche Applied Science, Penzberg, Germany). The protein concentration was determined using Bradford Protein Assay kit (Bio-Rad Laboratories, Inc., Hercules, CA, USA). Each lane was loaded with $0.05 \mu \mathrm{g}$ total protein. Proteins were separated by $10 \%$ SDS-PAGE and transferred onto polyvinylidene fluoride (PVDF) membranes. The PVDF membranes were blocked with 5\% non-fat milk for $1 \mathrm{~h}$ at room temperature, and incubated with the following primary antibodies: Anti-cleaved CASP3 (cat. no. 9661), cleaved CASP8 (cat. no. 9496), p53 (cat. no. 2527) (all 1:1,000; Cell Signaling Technology, Inc., Danvers, MA, USA), GAPDH (cat. no. 59540), TNFRSF10A (cat. no. 32255), TNFRSF10B (cat. no. 166624), BCL2 associated agonist of cell death (BAD; cat. no. 8044), BCL2 associated $\mathrm{X}$, apoptosis regulator apoptosis regulator (BAX; cat. no. 20067), BCL2 apoptosis regulator (BCL2; cat. no. 509) (all 1:1,000; Santa Cruz Biotechnology, Inc., Dallas, TX, USA) overnight at $4^{\circ} \mathrm{C}$. Horseradish peroxidase-conjugated goat anti-rabbit (cat. no. 7407) and goat anti-mouse (cat. no. 7076) secondary antibodies (all 1:10,000; Cell Signaling Technology, Inc.) were incubated with the membranes at room temperature for $2 \mathrm{~h}$. Bands were developed with the electrochemiluminescence detection reagent (GE Healthcare, Chicago, IL, USA). GAPDH was used as the loading control and Image $\mathbf{J}$ software (version 1.8.0, National Institutes of Health, Bethesda, MD, USA) was used to compare the relative band intensities.

RNA extraction and reverse transcription-quantitative polymerase chain reaction ( $R T-q P C R)$. HepG2 cells were incubated with DMEM for $48 \mathrm{~h}$, and total RNA was extracted using TRIzol ${ }^{\circledR}$ reagent (Invitrogen; Thermo Fisher Scientific, Inc.), according to the manufacturer's protocol. mRNA was reverse-transcribed to cDNA using the PrimeScript ${ }^{\mathrm{TM}}$ RT reagent kit (Takara Bio, Inc., Otsu, Japan), according to the manufacturer's protocol. For cDNA synthesis, the RT reactions were incubated at $37^{\circ} \mathrm{C}$ for $1 \mathrm{~h}$ and at $70^{\circ} \mathrm{C}$ for $15 \mathrm{~min}$. The sequences of the PCR primers used were as follows: TNFRSF10A forward: 5'-ACCTTCAAGTTTG TCGTCGTC-3'; TNFRSF10A reverse: 5'-CCAAAGGGCTAT GTTCCCATT-3'; TNFRSF10B forward: 5'-GCCCCACAA CAAAAGAGGTC-3'; TNFRSF10B reverse: 5'-AGGTCATTC CAGTGAGTGCTA-3'; GAPDH forward: 5'-ACAACTTTG GTATCGTGGAAGG-3'; GAPDH reverse: 5'-GCCATCACG CCACAGTTTC-3'. GAPDH gene was used as the reference gene. QuantiTect SYBR ${ }^{\circledR}$ Green RT-PCR kit (cat. no. 204245; Qiagen $\mathrm{GmbH}$, Hilden, Germany) was used to analyze relative gene expression. The thermocycling conditions were as follows: Initial denaturation at $95^{\circ} \mathrm{C}$ for $10 \mathrm{~min}$, followed by 40 cycles of $95^{\circ} \mathrm{C}$ for $15 \mathrm{sec}$ and $60^{\circ} \mathrm{C}$ for $60 \mathrm{sec}$, and a final elongation at $72^{\circ} \mathrm{C}$ for $15 \mathrm{sec}$.

Small interfering (siRNA) transfection. p53 siRNA (cat. no. sc-29435) and control siRNA (cat. no. sc-44238) were purchased from Santa Cruz Biotechnology, Inc. (Dallas, TX, USA). For the siRNA transfection, HepG2 cells were seeded in 6-well plates at $3 \times 10^{5}$ cells/well and cultured in an incubator at $37^{\circ} \mathrm{C}$ overnight. On the following day, $0.67 \mu \mathrm{g}$ siRNAs were mixed with Lipofectamine ${ }^{\circledR}$ RNAiMAX (Invitrogen; Thermo Fisher Scientific, Inc.) in Opti-MEM ${ }^{\mathrm{TM}}$ (Gibco; Thermo Fisher Scientific, Inc.) and incubated at room temperature for $15 \mathrm{~min}$; 

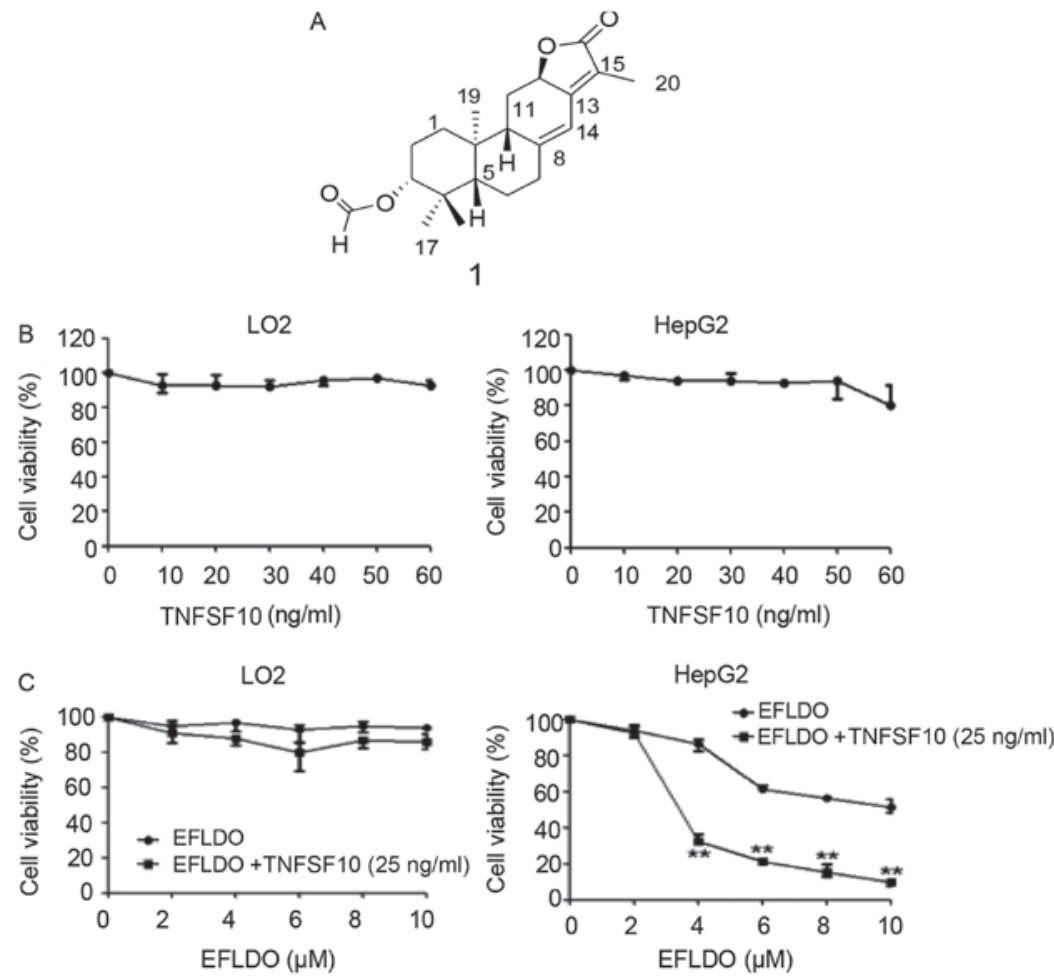

Figure 1. EFLDO increases TNFSF10-induced cell viability inhibition of HepG2 cells. (A) Chemical structure of EFLDO. (B) Non-cancerous normal liver LO2 cells and liver cancer HepG2 cells treated with TNFSF10 and with increasing concentrations of TNFSF10 for 24 h. Cell viability was measured using the MTT assay. (C) LO2 cells and HepG2 cells treated with TNFSF10 $(25 \mathrm{ng} / \mathrm{ml})$ and EFLDO for $24 \mathrm{~h}$. Cell viability was assessed using the MTT assay. ${ }^{* *}$ P $<0.01$ vs. respective EFLDO group. EFLDO, Ent-3 $\alpha$-formylabieta-8(14),13(15)-dien-16,12 $\beta$-olide; TNFSF10, tumor necrosis factor superfamily member 10 .

subsequently, the mixtures were added into the culture medium and incubated at $37^{\circ} \mathrm{C}$ for $24 \mathrm{~h}$ prior to further experimentation.

Statistical analysis. All data were analyzed using GraphPad Prism 6.0 (GraphPad Software, Inc., La Jolla, CA, USA). Data are presented as the mean \pm standard deviation. Comparisons between two groups and multiple groups were performed using Student's t-test and one-way analysis of variance followed by Newman-Keuls test, respectively. $\mathrm{P}<0.05$ was considered to indicate a statistically significant difference. All the experiments were performed $\geq 3$ times.

\section{Results}

Synergistic effects of EFLDO and TNFSF10 on HepG2 cell viability. The chemical structure of EFLDO is presented in Fig. 1A. The cytotoxicity of TNFSF10 at various concentrations $(10,20,30,40,50$ and $60 \mathrm{ng} / \mathrm{ml})$ on LO2 and HepG 2 cells was assessed using the MTT assay. The present MTT results suggested that TNFSF10 exhibited no obvious cytotoxicity in cells at concentrations $\leq 60 \mathrm{ng} / \mathrm{ml}$ (Fig. 1B).

Subsequently, the effects of various concentrations of EFLDO $(2,4,6,8$ and $10 \mu \mathrm{M})$ and $\operatorname{EFLDO}(2,4,6,8$ and $10 \mu \mathrm{M})+$ TNFSF10 $(25 \mathrm{ng} / \mathrm{ml})$ on cell viability were detected. The present results suggested that the cell viability of HepG2 cells significantly decreased following treatment with EFLDO, in a concentration-dependent manner. Notably, the cell viability of normal liver LO2 cells was unaffected by treatment with EFLDO (Fig. 1C). Additionally, the cell viability of HepG2 cells was significantly decreased following the administration of
EFLDO + TNFSF10 compared with administration of EFLDO alone. Furthermore, treatment with EFLDO $(3 \mu \mathrm{M})+$ TNFSF10 (25 ng/ml) exhibited a significant antiproliferative activity (data not shown). Therefore, $25 \mathrm{ng} / \mathrm{ml}$ was selected at the working concentration of TNFSF10 and $3 \mu \mathrm{M}$ was selected as the working concentration of EFLDO for further experiments.

EFLDO increases TNFSF10-induced cell apoptosis in Hep 2 cells. The potential of EFLDO in sensitizing HepG2 cells to TNFSF10-induced apoptosis was investigated. Flow cytometry results suggested that the apoptotic rate of HepG2 cells following treatment with EFLDO + TNFSF10 increased compared with the control, TNFSF10 and EFLDO groups (Fig. 2A). The apoptotic rates for HepG2 cells in the TNFSF10 and EFLDO + TNFSF10 group were 9.57 and $60.7 \%$, respectively (Fig. 2B). The present results suggested that the combination of EFLDO and TNFSF10 increased the effect of TNFSF10 in inducing the apoptosis of HepG2 cells.

EFLDO promotes TNFSF10-induced apoptosis of HepG2 cells in a caspase-dependent manner. Previous studies demonstrated that activation of CASP8 induced by TNFSF10 may activate CASP3 and CASP9 (12-14). To investigate the molecular mechanisms underlying cell apoptosis induced by treatment with EFLDO + TNFSF10, the activity levels of CASP8 and 3 in HepG2 cells treated with TNFSF10 and/or EFLDO were detected. The present results suggested that proteolytic cleavage of proCASP8 and proCASP3 increased following treatment with EFLDO + TNFSF10 compared with the control, TNFSF10 and EFLDO groups 
A
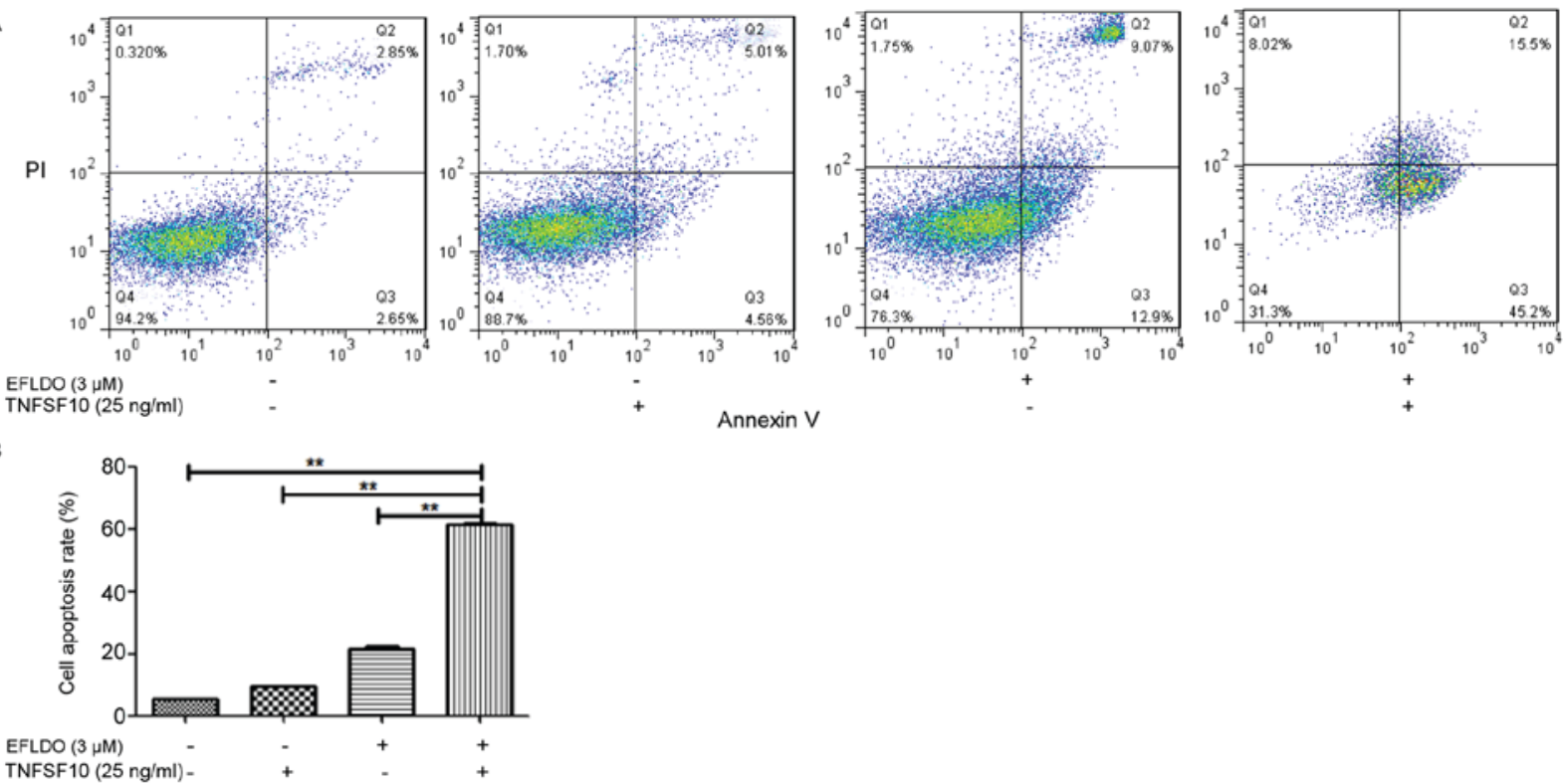

Figure 2. EFLDO increases TNFSF10-induced cell apoptosis. (A) HepG2 cells treated with EFLDO and/or TNFSF10 for 24 h. Flow cytometry was used to analyze the percentage of Annexin V/PI positive cells and the percentage of apoptotic cells was measured. (B) Statistical analysis of apoptotic rates. ${ }^{* *} \mathrm{P}<0.01$. EFLDO, Ent-3 $\alpha$-formylabieta-8(14),13(15)-dien-16,12ß-olide; TNFSF10, tumor necrosis factor superfamily member 10; PI, propidium iodide.
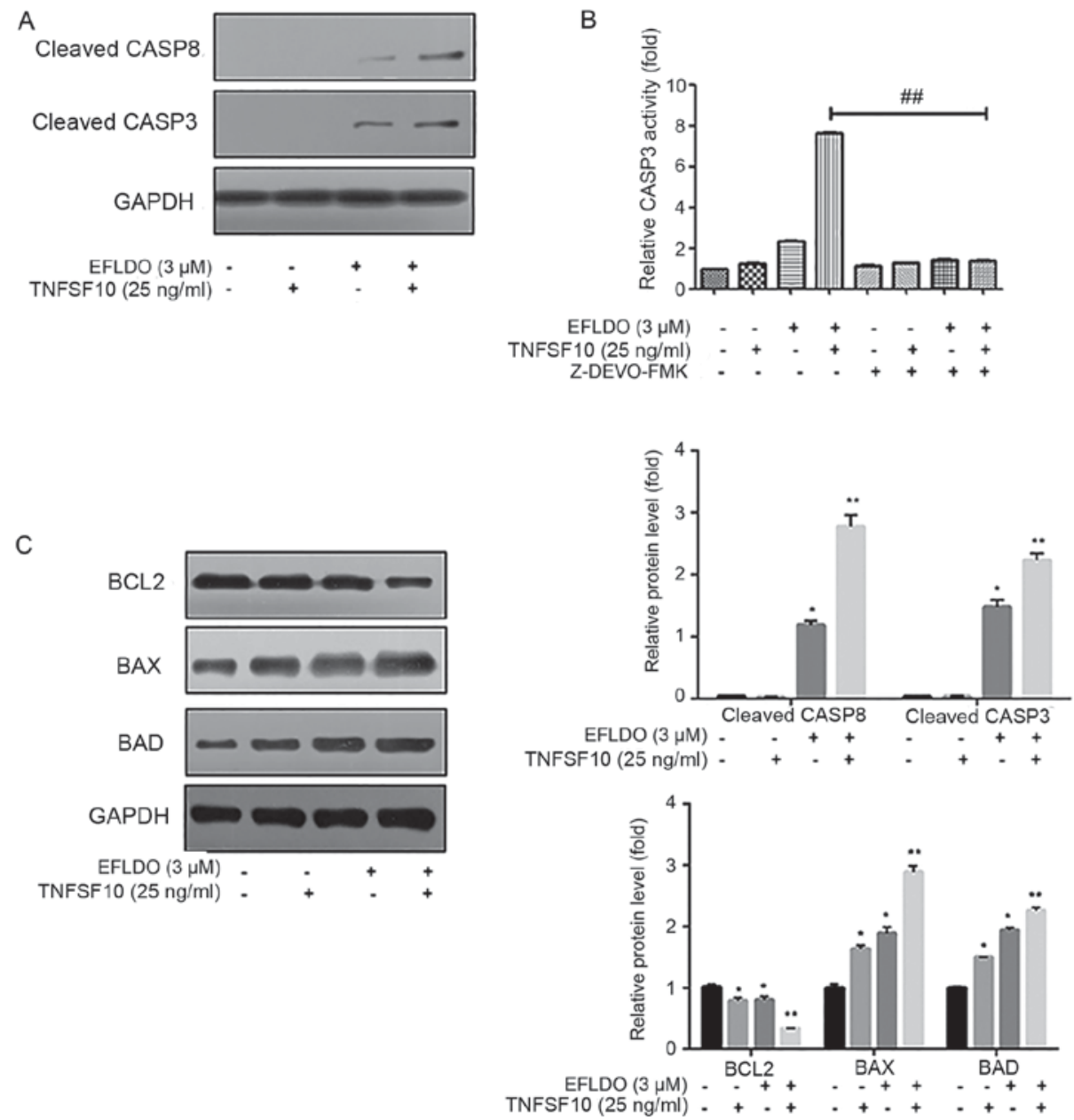

Figure 3. Treatment with EFLDO sensitizes HepG2 cells to TNFSF10-induced apoptosis via the caspase pathway. (A) Protein expression levels of cleaved CASP8 and CASP3 in HepG2 cells following treatment with EFLDO and/or TNFSF10. (B) Activity of CASP3 in liver cancer cells treated with EFLDO, TNFSF10 and/or caspase inhibitor z-DEVD-FMK. Data are presented as fold change relative to the mean CASP3 activity of the control group. ${ }^{\# \#<} \mathrm{P}<0.01$. (C) Protein expression levels of BLC2, BAX and BAD in HepG2 cells treated with EFLDO and/or TNFSF10. ${ }^{*} \mathrm{P}<0.05,{ }^{* * *} \mathrm{P}<0.01$ vs. respective control group. EFLDO, Ent-3 $\alpha$-formylabieta-8(14),13(15)-dien-16,12 $\beta$-olide; TNFSF10, tumor necrosis factor superfamily member 10; CASP, caspase; BCL2, BCL2 apoptosis regulator; BAD, BCL2 associated agonist of cell death; BAX, BCL2 associated X, apoptosis regulator. 

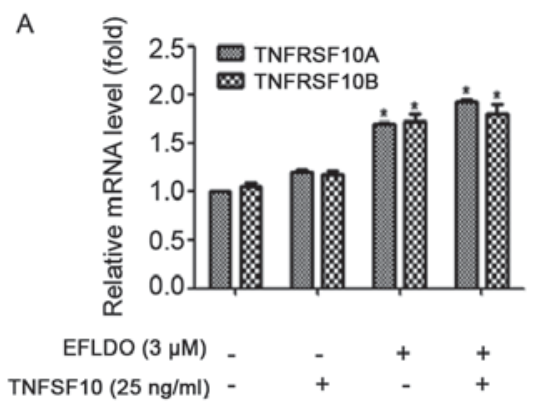
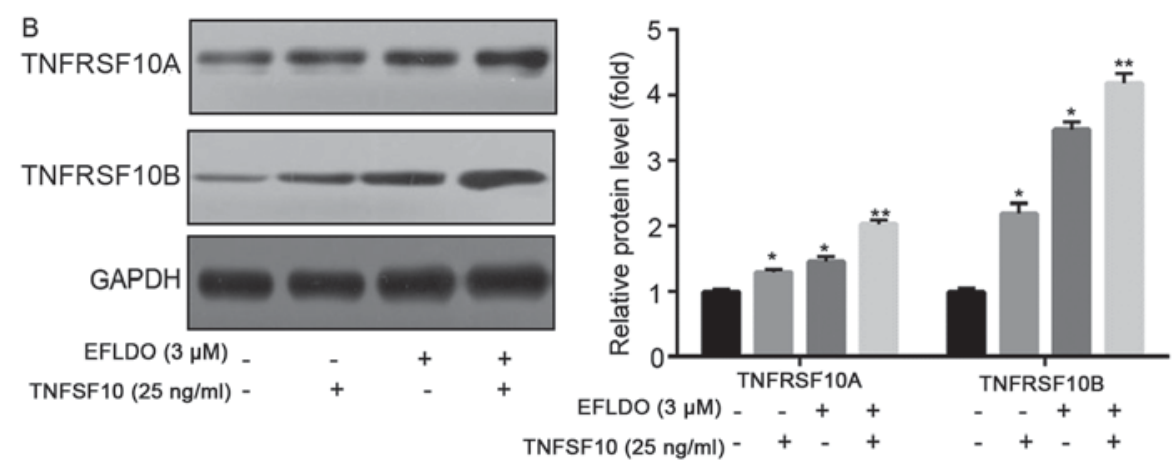

C

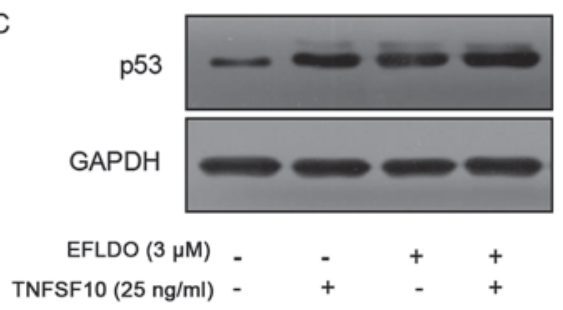

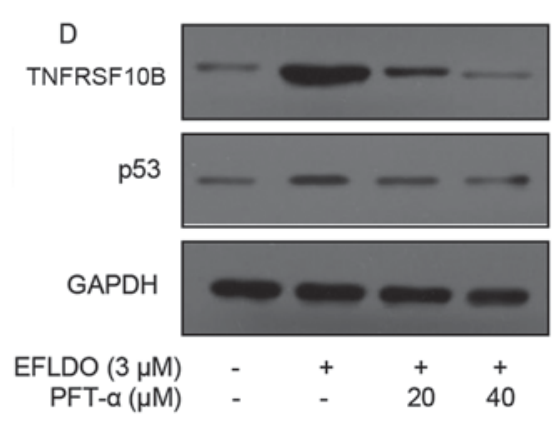

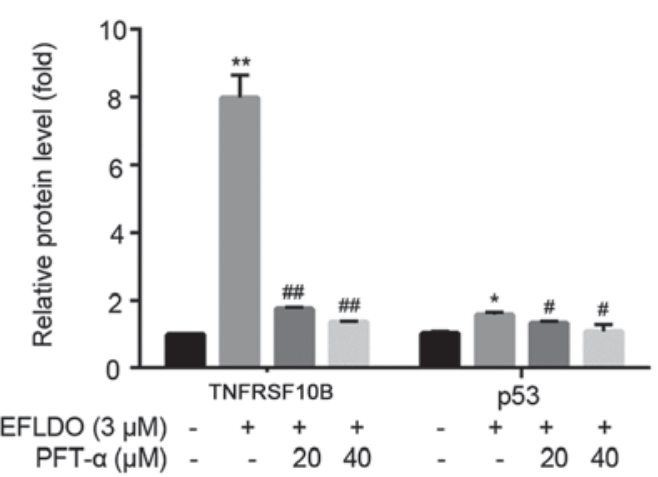

Figure 4. EFLDO treatment sensitizes liver cancer cells to TNFSF10-induced apoptosis by upregulating the protein expression levels of TNFRSF10A and TNFRSF10B in a p53-dependent manner. (A) HepG2 cells were treated with EFLDO and/or TNFSF10 for 24 h. Reverse transcription-quantitative polymerase chain reaction analysis was performed to detect the mRNA expression levels of TNFRSF10A and TNFRSF10B. In total, three independent experiments were performed. (B) Western blotting was performed to detect the protein expression levels of TNFRSF10A and TNFRSF10B. (C) Protein expression levels of p53 in HepG2 cells treated with EFLDO and/or TNFSF10. (D) Protein expression levels of TNFRSF10B and p53 in HepG2 cells treated with EFLDO, TNFSF10 and/or PFT- $\alpha$. "P $<0.05,{ }^{* * *} \mathrm{P}<0.01$ vs. respective control group. ${ }^{\#} \mathrm{P}<0.05,{ }^{\# \#} \mathrm{P}<0.01$ vs. respective EFLDO group. EFLDO, Ent-3 $\alpha$-for mylabieta-8(14),13(15)-dien-16,12ß-olide; TNFSF10, tumor necrosis factor superfamily member 10; TNFRSF10A, TNF receptor superfamily member 10a; TNFRSF10B, TNF receptor superfamily member $10 \mathrm{~b}$.

(Fig. 3A). CASP3 activity in the EFLDO + TNFSF10 group increased to 7.0 -fold compared with the control group, which was increased compared with TNFSF10 (0.7-fold) and EFLDO (1.4-fold) groups, and was significantly decreased following treatment with the caspase inhibitor z-DEVD-FMK (Fig. 3B). Subsequently, treatment with EFLDO + TNFSF10 significantly increased the protein expression levels of BCL2 associated agonist of cell death (BAD) and BAX, and decreased the protein expression level of BCL2 in HepG2 cells compared with the control, TNFSF10 and EFLDO groups (Fig. 3C). The present results suggested that EFLDO significantly sensitized HepG2 cells to TNFSF10-induced apoptosis in a caspase-dependent manner.

EFLDO sensitizes HepG2 cells to TNFSF10-induced cell apoptosis by increasing the expression levels of TNFRSF10A and TNFRSF10B. TNFRSF10A and TNFRSF10B (TNFSF10 receptors) were identified to be able to initiate the apoptotic cascade by binding to TNFSF10 (21). The present RT-qPCR and western blotting results suggested that treatment with EFLDO or EFLDO + TNFSF10 increased the mRNA (Fig. 4A) and protein expression levels (Fig. 4B) of TNFRSF10A and TNFRSF10B compared with the control group. A previous study observed that p53 may serve a role in regulating the functions of TNFRSF10A and TNFRSF10B (22). The present western blotting results suggested that, compared with the control group, the protein expression level of p53 was significantly increased following treatment with EFLDO or EFLDO + TNFSF10 (Fig. 4C). The effects of EFLDO on the protein expression levels of p53 and TNFRSF10B were inhibited by treatment with PFT- $\alpha$ (Fig. 4D). The present results suggested that treatment with EFLDO + TNFSF10 increased the protein expression levels of TNFRSF10A and TNFRSF10B by increasing the protein expression level of p53 in HepG2 cells. 
A

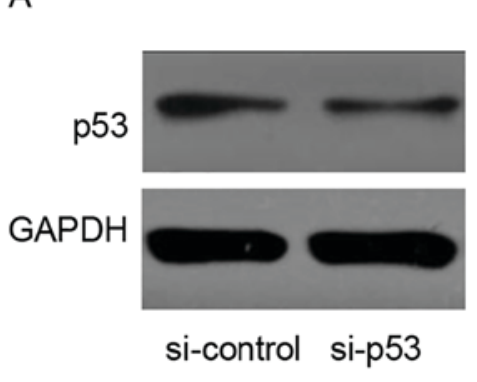

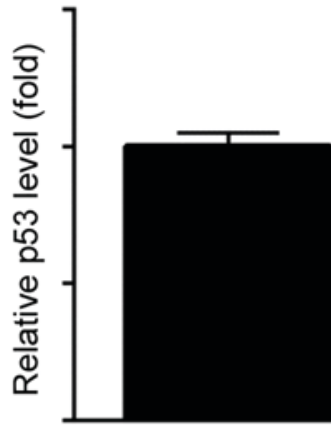

si-control

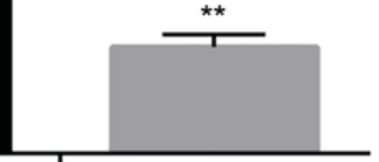

si-p53
B

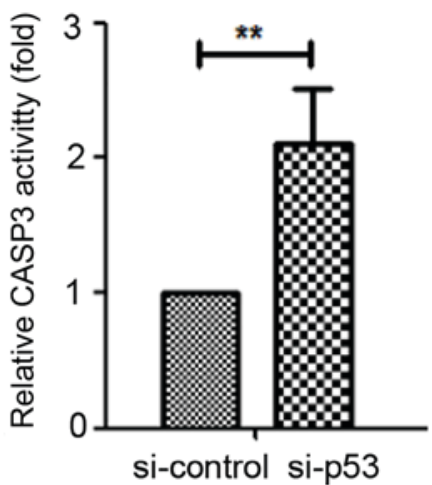

C
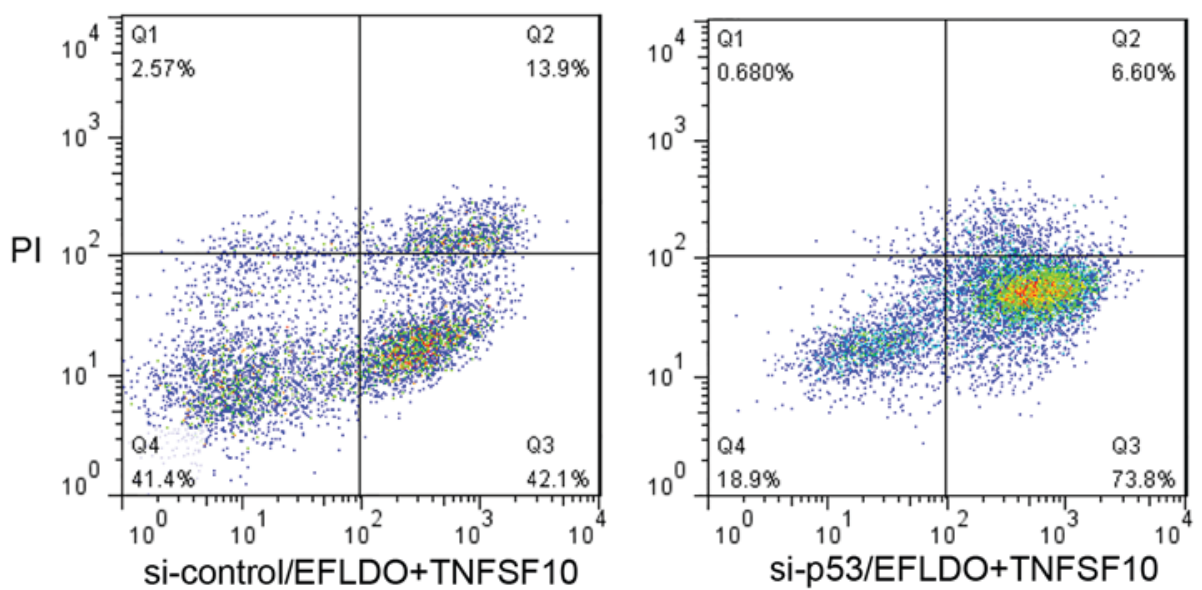

Annexin $\mathrm{V}$

Figure 5. EFLDO increases TNFSF10-induced cell apoptosis in a p53-dependent manner in HepG2 cells. (A) p53 was silenced using siRNA technology in HepG2 cells. (B) Activity of CASP3 was detected in HepG2 cells transfected with si-p53 or si-control and treated with the combination of EFLDO and TNFSF10 for $24 \mathrm{~h}$. Data are presented as a fold change relative to the mean CASP3 activity of the control group. (C) HepG2 cells were transfected with si-p53 and treated with the combination of EFLDO and TNFSF10. Following $24 \mathrm{~h}$, cells were harvested for flow cytometry analysis. ${ }^{* *} \mathrm{P}<0.01 \mathrm{vs}$. si-control. EFLDO, Ent-3 $\alpha$-formylabieta-8(14),13(15)-dien-16,12 $\beta$-olide; TNFSF10, tumor necrosis factor superfamily member 10; PI, propidium iodide; FITC, fluorescein isothiocyanate; si-, small interfering RNA; CASP, caspase.

EFLDO increases TNFSF10-induced cell apoptosis in a p53-dependent manner in HepG2 cells. To investigate the role of p53 on the effects of EFLDO in increasing TNFSF10-induced cell apoptosis, p53 was silenced by transfecting a siRNA targeting p53 (si-p53) in HepG2 cells (Fig. 5A). Knockdown of p53 significantly increased the activity of CASP3 in liver cancer cells (Fig. 5B). si-p53 significantly increased the apoptosis of HepG2 cells following treatment of TNFSF10 + EFLDO (Fig. 5C). The present results suggested that inhibition of p53 may increase EFLDO-induced TNFSF10 sensitivity in HepG2 cells.

\section{Discussion}

Previous studies in liver cancer demonstrated that the upregulation of antiapoptotic genes, and the increase in the protein expression levels of dysadherin and various cytokines, may serve roles in the negative outcome of certain therapies and in cancer metastasis (23-25). Combinatorial treatments were identified to exhibit an increased effectiveness compared with single-agent chemotherapy in various types of cancer, including liver cancer (26). Nevertheless, the efficacy of chemotherapy to treat cancer may be decreased by the occurrence of drug resistance. Therefore, the identification of novel therapies is required to improve the treatment of patients with liver cancer.

Due to its strong antiproliferative activity in MCF-7 and NCI-H460 cells (17), EFLDO has attracted the interest of various research studies (18). However, the effects of the combination of EFLDO with additional anticancer drugs in liver cancer remain unknown.

The present results suggested that TNFSF10 did not induce cytotoxicity. By contrast, the combination of EFLDO and TNFSF10 presented a synergistic effect in suppressing cell viability of HepG2 cells; however, this combinatorial treatment did not affect the viability of non-cancerous normal liver LO2 cells. Subsequently, EFLDO was identified to sensitize HepG2 cells to TNFSF10-induced apoptosis. The present results suggested that apoptotic rates of HepG2 cells increased significantly following the cotreatment with TNFSF10 and EFLDO compared with single-treatment with TNSFS10 or EFLDO, indicating a potential synergistic effect between TNFSF10 and EFLDO in inducing apoptosis in liver cancer cells.

Furthermore, the activities of CASP8 and 3 following treatment with TNFSF10 and/or EFLDO were detected to investigate the molecular mechanisms underlying cell apoptosis induced by the combined treatment with EFLDO and TNFSF10. The present results suggested that liver cancer 
cell apoptosis was induced by EFLDO + TNFSF10 via the activation of CASP3 and CASP8. Treatment with EFLDO and TNSFS10 induced the upregulation of pro-apoptotic molecules, including BAD and BAX, and the downregulation of the anti-apoptotic factor BCL2. The present results suggested that EFLDO may promote TNFSF10-induced liver cancer cell apoptosis in a caspase-dependent manner.

p53 was previously identified to serve a role in regulating the function of TNFRSF10A and TNFRSF10B (22). p53 is able to inhibit cell proliferation by inducing cell apoptosis and cell cycle arrest in response to cellular stresses (27-29). The present results suggested that the synergistic effects between EFLDO and TNFSF10 in increasing the protein expression level of TNFRSF10B may be due to the upregulation of p53, since the protein expression level of TNFRSF10B was decreased following treatment with the $\mathrm{p} 53$ inhibitor PFT- $\alpha$. In addition, knockdown of p53 increased the activity of CASP3 and increased cell apoptosis in HepG2 cells, suggesting that p53 may regulate the synergistic effects between EFLDO and TNFSF10 in liver cancer cells. However, treatment with EFLDO and TNFSF10 led to an increase in the expression level of p53, in contrast with the results of p53 knockdown. p53 may be involved in various pathways regulating HepG2 cells, and further studies are required to confirm the present results.

In conclusion, combinatorial treatment with TNFSF10 and EFLDO induced cell apoptosis in HepG2 cells by promoting the caspase pathway, suggesting a potential therapeutic effect of the combination of EFLDO and TNFSF10 in treating liver cancer.

\section{Acknowledgements}

The authors would like to thank The Pharmaceutical Engineering Department of Southeast University for technical support of this study.

\section{Funding}

The project was funded by the priority academic program development of Jiangsu Higher Education Institutions (grant no. 1107047002), and the Key Laboratory for Chemistry and Molecular Engineering of Medicinal Resources (Guangxi Normal University; grant nos. CMEMR2016-B06 to ZXL).

\section{Availability of data and materials}

The datasets used and/or analyzed during the current study are available from the corresponding author on reasonable request.

\section{Authors' contributions}

YQ acquired the data, analyzed and interpreted the data, drafted the manuscript and revised it critically. ZL designed and conceived the study and revised the manuscript. XW, JZ and CL performed the experiments. All authors read and approved the final manuscript.

\section{Ethics approval and consent to participate}

Not applicable.

\section{Patient consent for publication}

Not applicable.

\section{Competing interests}

The authors declare that they have no competing interests.

\section{References}

1. Takada Y, Tohyama T and Watanabe J: Biological markers of hepatocellular carcinoma for use as selection criteria in liver transplantation. J Hepatobiliary Pancreat Sci 22: 279-286, 2015.

2. Ling D, Xia H, Park W, Hackett MJ, Song C, Na K, Hui KM and Hyeon T: pH-sensitive nanoformulated triptolide as a targeted therapeutic strategy for hepatocellular carcinoma. ACS Nano 8: 8027-8039, 2014

3. Kassebaum NJ, Bertozzi-Villa A, Coggeshall MS, Shackelford KA, Steiner C, Heuton KR, Gonzalez-Medina D, Barber R, Huynh C, Dicker D, et al: Global, regional, and national levels and causes of maternal mortality during 1990-2013: A systematic analysis for the Global burden of disease study 2013. Lancet 384: 980-1004, 2014.

4. Alsaied OA, Sangwan V, Banerjee S, Krosch TC, Chugh R, Saluja A, Vickers SM and Jensen EH: Sorafenib and triptolide as combination therapy for hepatocellular carcinoma. Surgery 156: 270-279, 2014.

5. Chen W, Zhang Y, Li Y, Zhang J, Zhang T, Fu B, Zhang Q and Jiang N: Constitutive expression of Wnt $/ \beta$-catenin target genes promotes proliferation and invasion of liver cancer stem cells. Mol Med Rep 13: 3466-3474, 2016.

6. He S, Chen Y, Chen XP, Zhang WG, Wang HP, Zhao YZ and Wang SF: Antitumor effects of soluble TRAIL in human hepatocellular carcinoma. J Huazhong Univ Sci Technolog Med Sci 25: 51-54, 2005.

7. He SQ, Rehman H, Gong MG, Zhao YZ, Huang ZY, Li CH, Zhang WG and Chen XP: Inhibiting survivin expression enhances TRAIL-induced tumoricidal activity in human hepatocellular carcinoma via cell cycle arrest. Cancer Biol Ther 6: 1247-1257, 2007.

8. Zhang Y,MaH,Zhang J,Liu S,Liu Y and Zheng D: AAV-mediated TRAIL gene expression driven by hTERT promoter suppressed human hepatocellular carcinoma growth in mice. Life Sci 82: 1154-1161, 2008.

9. Zhang Y, Qu ZH, Cui M, Guo C, Zhang XM, Ma CH and Sun WS: Combined endostatin and TRAIL gene transfer suppresses human hepatocellular carcinoma growth and angiogenesis in nude mice. Cancer Biol Ther 8: 466-473, 2009.

10. Zhang B, Shan H, Li D, Zhu KS, Jiang ZB and Huang MS: Cisplatin sensitizes human hepatocellular carcinoma cells, but not hepatocytes and mesenchymal stem cells, to TRAIL within a therapeutic window partially depending on the upregulation of DR5. Oncol Rep 25: 461-468, 2011.

11. Lee JY, Jung KH, Morgan MJ, Kang YR, Lee HS, Koo GB, Hong SS, Kwon SW and Kim YS: Sensitization of TRAIL-induced cell death by 20(S)-ginsenoside Rg3 via CHOP-mediated DR5 upregulation in human hepatocellular carcinoma cells. Mol Cancer Ther 12: 274-285, 2013.

12. Okano H, Shiraki K, Inoue H, Kawakita T, Yamanaka T, Deguchi M, Sugimoto K, Sakai T, Ohmori S, Fujikawa K, et al: Cellular FLICE/caspase-8-inhibitory protein as a principal regulator of cell death and survival in human hepatocellular carcinoma. Lab Invest 83: 1033-1043, 2003.

13. Ganten TM, Haas TL, Sykoraet J, Stahl H, Sprick MR, Fas SC, Krueger A, Weigand MA, Grosse-Wilde A, Stremmel W, et al: Enhanced caspase- 8 recruitment to and activation at the DISC is critical for sensitisation of human hepatocellular carcinoma cells to TRAIL-induced apoptosis by chemotherapeutic drugs. Cell Death Differ 11 (Suppl 1): S86-S96, 2004.

14. Su CL, Wu CJ, Chen FN, Wang BJ, Sheu SR and Won SJ: Supernatant of bacterial fermented soybean induces apoptosis of human hepatocellular carcinoma Hep 3B cells via activation of caspase 8 and mitochondria. Food Chem Toxicol 45: 303-314, 2007. 
15. Youness ER, El Nemr M, Oraby FS, Ahmed NM, Moghni MA, Aly HF and Ahmed HH: Evaluation of apoptotic marker Bcl2, CD4+, human hepatocyte growth factor and metalloproteinase-9 as tumor markers for patients with hepatocellular carcinoma. Indian J Clin Biochem 29: 351-356, 2014.

16. Zhang L and Fang B: Mechanisms of resistance to TRAIL-induced apoptosis in cancer. Cancer Gene Ther 12: 228-237, 2005.

17. Liu C, Liao ZX, Liu SJ, Qu YB and Wang HS: Two new derivatives from Euphorbia lunulata bge and their anti-proliferation activities. Fitoterapia 96: 33-38, 2014.

18. Qu YB, Liao ZX, Liu C, Wang XZ and Zhang J: EFLDO induces apoptosis in hepatic cancer cells by caspase activation in vitro and suppresses tumor growth in vivo. Biomed Pharmacother 100: 407-416, 2018

19. López-Terrada D, Cheung SW, Finegold MJ and Knowles BB: HepG2 is a hepatoblastoma-derived cell line. Hum Pathol 40 $1512-1515,2009$

20. Twentyman P and Luscombe M: A study of some variables in a tetrazolium dye (MTT) based assay for cell growth and chemosensitivity. Br J Cancer 56: 279-285, 1987.

21. Fossati S, Ghiso J and Rostagno A: TRAIL death receptors DR4 and DR5 mediate cerebral microvascular endothelial cell apoptosis induced by oligomeric Alzheimer's A $\beta$. Cell Death Dis 3 : e321, 2012.

22. Tomasetti M, Andera L, Alleva R, Borghi B, Neuzil J and Procopio A: Alpha-tocopheryl succinate induces DR4 and DR5 expression by a 53 -dependent route: Implication for sensitisation of resistant cancer cells to TRAIL apoptosis. FEBS Lett 580: 1925-1931, 2006.
23. Dalerba P, Dylla SJ, Park IK, Liu R, Wang X, Cho RW, Hoey T, Gurney A, Huang EH, Simeone DM, et al: Phenotypic character-ization of human colorectal cancer stem cells. Proc Nat Acad Sci USA 104: 10158-10163, 2007.

24. Ino Y, Gotoh M, Sakamoto M, Tsukagoshi K and Hirohashi S: Dysadherin, a cancer-associated cell membrane glycoprotein, down-regulates E-cadherin and promotes metastasis. Proc Natl Acad Sci USA 99: 365-370, 2002.

25. Nam JS, Hirohashi S and Wakefield LM: Dysadherin: A new player in cancer progression. Cancer Lett 255: 161-169, 2007.

26. El-Shemi AG, Ashshi AM, Na Y, Li YM, Al-Allaf FA, Oh E, Jung BK and Yun CO: Combined therapy with oncolytic adenoviruses encoding TRAIL and IL-12 genes markedly suppressed human hepatocellular carcinoma both in vitro and in an orthotopic transplanted mouse model. J Exp Clin Cancer Res 35: 74, 2016.

27. Chiu CT, Yeh TS, Hsu JC and Chen M: Expression of Bcl-2 family modulated through p53-dependent pathway in human hepatocellular carcinoma. Dig Dis Sci 48: 670-676, 2003.

28. Franklin DA, He Y, Leslie PL, Tikunov AP, Fenger N Macdonal JM and Zhang Y: p53 coordinates DNA repair with nucleotide synthesis by suppressing PFKFB3 expression and promoting the pentose phosphate pathway. Sci Rep 6: 38067 , 2016.

29. Romano FJ, Rossetti S, Conteduca V, Schepisi G, Cavaliere C, Di Franco R, La Mantia E, Castaldo L, Nocerino F, Ametrano G, et al: Role of DNA repair machinery and p53 in the testicular germ cell cancer: A review. Oncotarget 7: 85641-85649, 2016. 